\title{
A Case of Acute Pemphigus Vulgaris Relapses Associated with Cocaine Use and Review of the Literature
}

\author{
Omar Jiménez-Zarazúa • Andrés Guzmán-Ramírez • Lourdes N. Vélez-Ramírez • \\ Jesús A. López-García · Leticia Casimiro-Guzmán · Jaime D. Mondragón
}

Received: September 19, 2018 / Published online: November 10, 2018

(C) The Author(s) 2018

\section{ABSTRACT}

Pemphigus is a bullous autoimmune disease that affects the skin and mucous membranes. It is very difficult to establish the etiology and the triggering factors that influence reactivations in pemphigus vulgaris (PV). The case of a 33-yearold male with chronic history of intranasal cocaine consumption is presented in this report. We present the clinical case of the patient, followed for a total of 86 weeks, with ten relapses secondary to probable cocaine use.

Enhanced Digital Features To view enhanced digital features for this article go to https://doi.org/10.6084/ m9.figshare.7291892.

Electronic supplementary material The online version of this article (https://doi.org/10.1007/s13555018-0271-0) contains supplementary material, which is available to authorized users.

O. Jiménez-Zarazúa

Department of Internal Medicine, Hospital General León, León, Guanajuato, Mexico

O. Jiménez-Zarazúa

Department of Medicine and Nutrition, University of Guanajuato, León, Guanajuato, Mexico

A. Guzmán-Ramírez

Department of Dermatology, Hospital General

León, León, Guanajuato, Mexico

L. N. Vélez-Ramírez

Department of Radiology, Hospital General León, León, Guanajuato, Mexico
The patient was admitted to the emergency department after presenting polymorphic dermatosis characterized by blisters, vesicles, and excoriations extending from the oral cavity to the thorax, and to the inguinal and genital regions, affecting approximately $35 \%$ of the body surface area with a score of 56 on the Pemphigus Skin Disorder Index. Skin biopsies were compatible with PV diagnosis. The patient had clinical improvement with a combination of methylprednisolone $500 \mathrm{mg}$ intravenously (IV) and cyclophosphamide $500 \mathrm{mg}$ IV every 15 days, along with prednisone $50 \mathrm{mg}$ orally (PO) q24 h and mycophenolic acid $500 \mathrm{mg}$ PO q6 h. Persistent cocaine use is highly likely to be the factor triggering lesion reactivation and responsible for the torpid evolution. We cannot definitively conclude whether the change from azathioprine to mycophenolic acid after the tenth relapse was the adjuvant medication

\section{J. A. López-García}

Head of the Department of Internal Medicine and Rheumatology, Hospital General León, León, Guanajuato, Mexico

L. Casimiro-Guzmán Department of Pathology, Hospital General León, León, Guanajuato, Mexico

J. D. Mondragón ( $\bowtie)$

Department of Neurology, Alzheimer Research Center Groningen, University of Groningen,

University Medical Center Groningen, Groningen, The Netherlands

e-mail: j.d.mondragon.uribe@umcg.nl 
responsible for the end of the consolidation phase and complete remission on therapy. This case study could potentially serve as a guide for management of patients who continuously persist with cocaine use, leading to a clinical picture refractory to multiple therapeutic schemes.

Keywords: Acantholytic lesion; Cocaine; Corticosteroid therapy; Mycophenolic acid; Pemphigus vulgaris; Relapse

\section{INTRODUCTION}

Pemphigus is a rare blistering disease that affects the skin and mucous membranes. It is an autoimmune disease characterized by production of pathogenic autoantibodies directed against desmosome proteins of keratinocytes in the epidermis [1]. There are two basic forms of pemphigus, viz. pemphigus vulgaris (PV) and pemphigus foliaceus (PF). The etiological factors associated with pemphigus are blunt trauma, ionizing radiation, stress, cocaine use, herpes simplex virus, Epstein-Barr virus, cytomegalovirus, and medication (e.g., hormones, vaccines, nonsteroidal antiinflammatory drugs) [2-5].

In PV, blisters develop above the stratum basale and are associated with autoantibodies against desmoglein (Dsg)3 (i.e., a cell surface adhesion molecule of keratinocytes). Meanwhile, in PF, blisters form under the stratum corneum and are associated with autoantibodies against Dsg1 [2]. PV diagnosis requires the following: (1) clinically compatible presentation, (2) lesions histopathologically compatible with direct immunofluorescence microscopy of perilesional skin, and (3) serological detection of autoantibodies against the surface of epithelial cells by indirect immunofluorescence microscopy and/or enzyme-linked immunosorbent assay (ELISA) [6]. Among the differential diagnoses for PV are PF, pemphigus erythematosus, pemphigus vegetans, and paraneoplastic pemphigus. Other dermatologic lesions with similar morphology include herpes simplex, bullous pemphigoid, dermatitis herpetiformis, erythema multiforme, and lichen planus [7]. We present the clinical case of a patient with ten relapses, probably secondary to cocaine use. The patient's evolution was torpid and refractory to first-line treatment, despite reported adequate adherence to treatment and without presenting other triggering factors. This is the first case reported in literature in which a patient with multiple relapses secondary to continuous cocaine use is refractory to multiple therapeutic schemes, but ultimately has complete remission after suspension of cocaine use and establishment of an effective therapeutic scheme. Approval from the ethical committee was not required due to the nature of this case report. Abiding by the 1964 Declaration of Helsinki and its later amendments or comparable ethical standards, patient anonymity was guaranteed. Upon hospital admission, the patient signed an informed consent form permitting use of their clinical file information for didactic and research purposes.

\section{CLINICAL PRESENTATION}

A 33-year-old male arrived at the emergency department, presenting polymorphic-type dermatosis with 1 week of evolution, characterized by blisters, vesicles, and excoriations on the scalp and oral cavity that spread to the thorax (anterior thorax impetiginized and posterior thorax denuded; Fig. 1), extending to the inguinal and genital area (sparing the penis and glans). Among the patient's personal history, he reported long-lasting alcohol abuse and drug addiction, consisting of daily inhalation of approximately $200 \mathrm{mg}$ cocaine during the previous 2 years. Cocaine use was suspended hours prior to hospital admission. The patient's family history included a father and two second-degree relatives with arterial hypertension under treatment. The patient had no history of skin, hematologic, autoimmune, or degenerative diseases or neoplasms.

The integumentary system suffered from dermal lesions (vide supra) affecting $35 \%$ of the total body surface (BSA) with a score of 56 on the Pemphigus Skin Disorder Index (PDAI). Thoracic, abdominal, and genital lesions were 

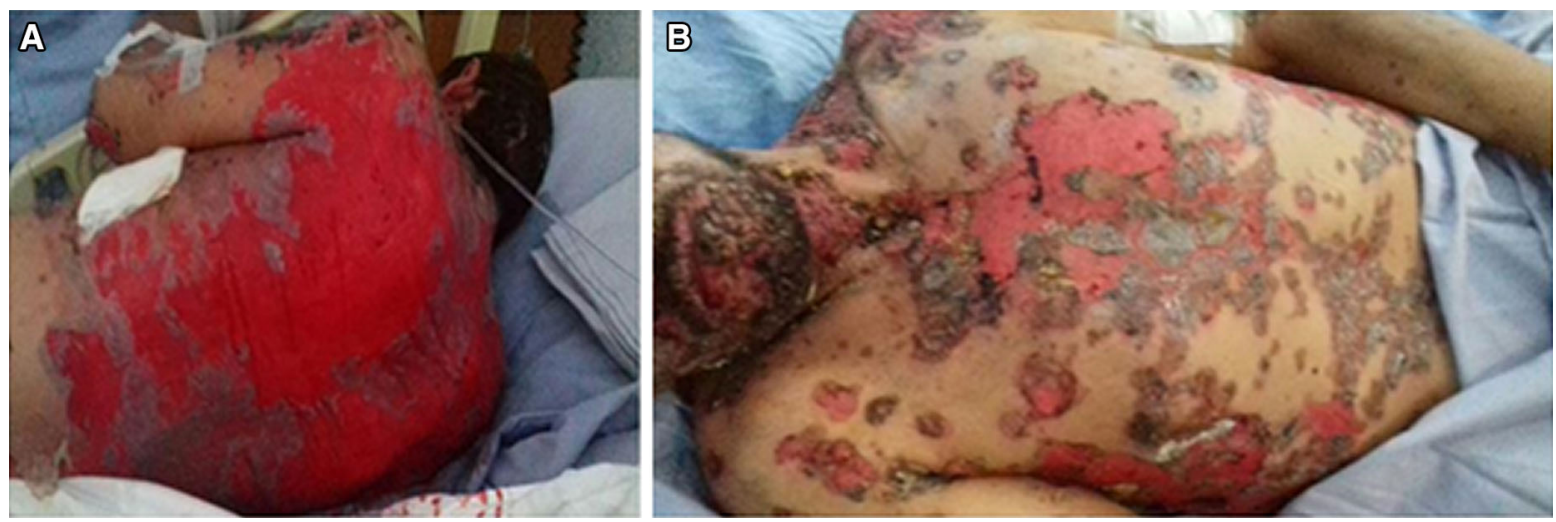

Fig. 1 a Photograph of posterior thorax. Active lesions (i.e., blisters, vesicles, and excoriations) with denuded regions of the back. b Photograph of face and anterior thorax. Impetiginized anterior thorax, scalp, and oral cavity, spreading to the thorax

positive for the Nikolsky sign at the edges, while the lower extremities presented blisters in healing phase. Clarithromycin, acyclovir, and nonsteroidal antiinflammatory drugs (NSAIDs) were indicated during the first week, without presenting clinical improvement. Upon admission, the patient had the following vital signs: blood pressure $110 / 80 \mathrm{mmHg}$, heart rate $90 \mathrm{bpm}$, respiratory rate $20 \mathrm{rpm}$, temperature $37.5^{\circ} \mathrm{C}$, weight $65 \mathrm{~kg}$, height $167 \mathrm{~cm}$, body mass index $23.1 \mathrm{~kg} / \mathrm{m}^{2}$.

During hospitalization, the patient had intermittent fever without time predominance. Laboratory results at admission are presented in Supplementary Table 1. Due to personal history of drug use, the following tests were requested: hepatitis B virus, hepatitis C virus, human immunodeficiency virus, and urinalysis for benzodiazepines, barbiturates, cannabis, cocaine, methamphetamines, and opiates; all results except urinalysis for cocaine metabolites were reported as negative. In search of an autoimmune etiology, the following tests were requested: anti-double-stranded deoxyribonucleic acid $(<0.5 \mathrm{U} / \mathrm{mL})$ and antinuclear antibody $(6.58 \mathrm{U} / \mathrm{mL})$; both reported as negative (Supplementary Table 2). Twenty-four hours after admission, skin biopsy was performed on the anterior thorax, with a suprabasal bullous lesion and acantholysis, compatible with PV (Fig. 2). Treatment was initiated with methylprednisolone intravenously (IV), azathioprine and prednisone orally (Table 1). Refer to the supplemental material for a detailed description of the case presentation and evolution.

\section{CLINICAL EVOLUTION}

The patient had torpid clinical evolution with multiple relapses (Fig. 3). The management strategy for this patient can be divided into six phases (Table 1). The first phase was performed when the patient was hospitalized. The second phase lasted only 1 month, while the third phase 5 months, the fourth phase 5 months, and the fifth phase 6 months; the final phase (i.e., until the time of manuscript preparation) included maintenance therapy until the patient achieved complete remission off therapy. In the absence of rituximab and intravenous immunoglobulin (IVIg) in our hospital, considering the therapeutic effectiveness of the schemes used, slight modifications of previous therapeutic algorithms were made and implemented. Full-body computerized tomography (CT) scan was performed to rule out paraneoplastic pemphigus, reporting negative result for neoplasia (Fig. 4).

Cocaine consumption was corroborated after each relapse despite alleged suspension via urinalyses for cocaine and its metabolites (Supplementary Table 2). The patient was first given information about the possible complications of chronic and acute cocaine use, then referred to the social work department for provision of information about support groups and nonprofit organizations dedicated to addiction management. Finally, 6 months after the change from azathioprine to mycophenolic acid, the patient achieved complete PV 


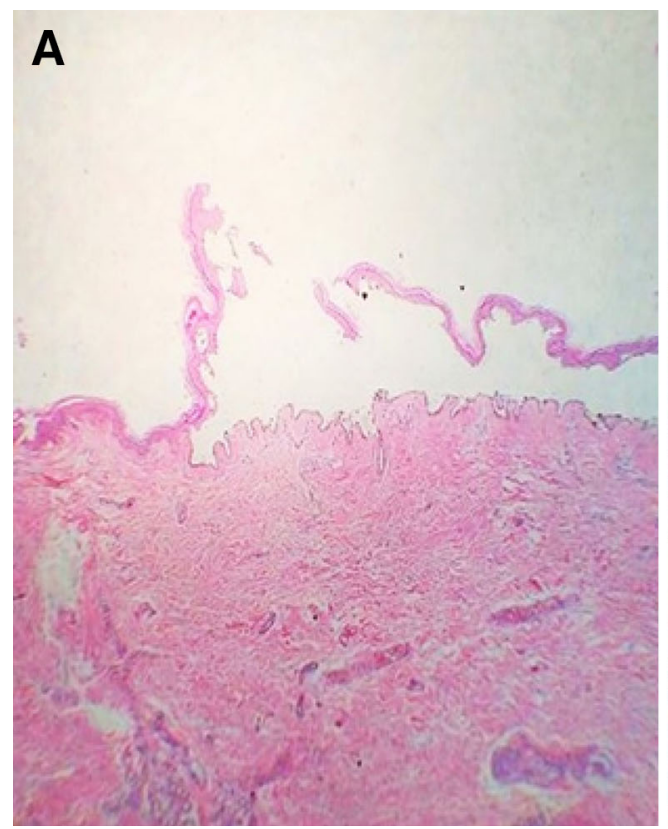

Fig. 2 a Histopathology of skin biopsy, 50×, hematoxylin and eosin staining. Presence of suprabasal bullous lesion. b Histopathology of skin biopsy, $100 \times$, hematoxylin and

remission (Fig. 5) and was followed on a monthly basis with management therapy with only prednisone $25 \mathrm{mg}$ per os (PO) q24 h. The patient was free of any PV relapses at the time of preparation of this manuscript, after 3 months with the maintenance therapeutic scheme of prednisone $25 \mathrm{mg}$ PO q24 h.

\section{DISCUSSION}

To the best of the authors' knowledge, this is the first report in literature of a case in which a patient with multiple relapses secondary to continuous cocaine use is refractory to multiple therapeutic schemes, but ultimately achieves complete remission after suspending cocaine use and establishing an effective therapeutic scheme. The patient presented in this case was followed for a total of 86 weeks, having a total of ten relapses. The patient had relapses despite good therapeutic adherence; nonetheless, the patient continued cocaine consumption without reducing the frequency or amount consumed for over 14 months after initiation of therapeutic management. The therapeutic

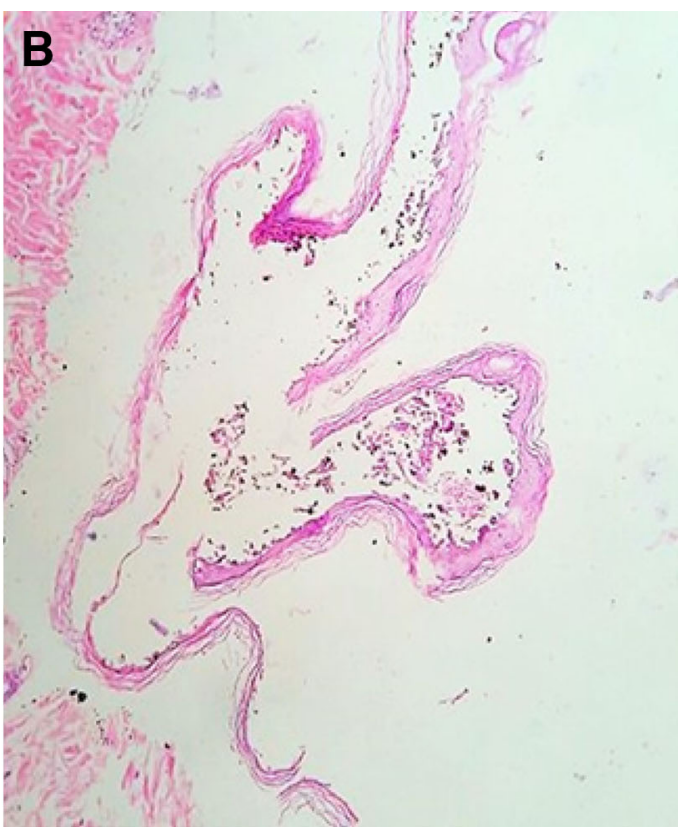

eosin staining. Presence of acantholytic cells within a bullous lesion with detachment of the rest of the epidermis

management was determined by two factors: first medication availability (or lack of access to rituximab and IVIg in our hospital), and secondly the risk-benefit balance associated with use of glucocorticoids and the clinical evolution.

The optimal therapeutic strategy for PV and $\mathrm{PF}$ is not known. Treatment of pemphigus is through use of glucocorticoids, reducing mortality from $75 \%$ to less than $10 \%$ [8]. Multiple treatment modalities and regimens are available, making the choice of treatment complex [9]. The management of this patient can be divided into six phases. During the first phase, the patient was administered three IV boluses of methylprednisolone (i.e., $3 \mathrm{~g}$ ) due to the severity of the clinical picture and to control the dermatosis immediately. Use of high glucocorticoid doses has been reported previously, with administration of methylprednisolone IV (i.e., $10-20 \mathrm{mg} / \mathrm{kg}$ or $250-1000 \mathrm{mg} /$ day) or equivalent doses of dexamethasone for up to five consecutive days [10]. The theoretical basis of use of glucocorticoid boluses is to achieve faster and more efficient control of the disease compared with conventional oral glucocorticoid 
Table 1 Clinical evolution

\begin{tabular}{|c|c|c|c|c|c|c|}
\hline $\begin{array}{l}\text { Treatment } \\
\text { phase }\end{array}$ & $\begin{array}{l}\text { Time } \\
\text { in } \\
\text { weeks }\end{array}$ & $\begin{array}{l}\text { Total body surface } \\
\text { with active lesions (\%) }\end{array}$ & $\begin{array}{l}\text { Pemphigus Skin } \\
\text { Disorder Index }\end{array}$ & Event & Treatment & $\begin{array}{l}\text { Frequency of } \\
\text { IV treatment }\end{array}$ \\
\hline 1 & 0 & 35 & 56 & Admission & $\mathrm{M}_{0}, \mathrm{P}_{0}, \mathrm{~A}_{0}$ & Once \\
\hline 2 & 2 & 24 & 19 & Discharge & $\mathrm{M}, \mathrm{P}, \mathrm{A}$ & Monthly \\
\hline 3 & 4 & 15 & 21 & 1st relapse & $\mathrm{M}, \mathrm{C}, \mathrm{P}, \mathrm{A}$ & Monthly \\
\hline 3 & 5 & 9 & 12 & 2nd discharge & $\mathrm{M}, \mathrm{C}, \mathrm{P}, \mathrm{A}$ & Monthly \\
\hline 3 & 6 & 15 & 12 & 2 nd relapse & $\mathrm{M}, \mathrm{C}, \mathrm{P}, \mathrm{A}$ & Monthly \\
\hline 3 & 9 & 12 & 11 & 3rd relapse & $\mathrm{M}, \mathrm{C}, \mathrm{P}, \mathrm{A}$ & Monthly \\
\hline 3 & 11 & 15 & 19 & 4th relapse & $\mathrm{M}, \mathrm{C}, \mathrm{P}, \mathrm{A}$ & Monthly \\
\hline 3 & 15 & 12 & 17 & 5th relapse & $\mathrm{M}, \mathrm{C}, \mathrm{P}, \mathrm{A}$ & Monthly \\
\hline 3 & 20 & 15 & 16 & 6th relapse & $\mathrm{M}, \mathrm{C}, \mathrm{P}, \mathrm{A}$ & Monthly \\
\hline 3 & 24 & 12 & 14 & 7th relapse & $\mathrm{M}, \mathrm{C}, \mathrm{P}, \mathrm{A}$ & Monthly \\
\hline 3 & 26 & 12 & 12 & 8th relapse & $\mathrm{M}, \mathrm{C}, \mathrm{P}, \mathrm{A}$ & Monthly \\
\hline 4 & 31 & 10 & 7 & 9th relapse & $\mathrm{M}, \mathrm{C}, \mathrm{P}, \mathrm{A}$ & Bimonthly \\
\hline 4 & 48 & 15 & 16 & $\begin{array}{l}\text { 10th relapse and } \\
\text { change of } \\
\text { treatment }\end{array}$ & $\mathrm{M}, \mathrm{C}, \mathrm{P}, \mathrm{A}$ & Bimonthly \\
\hline 5 & 59 & 3 & 7 & $\begin{array}{l}\text { Suspension of } \\
\text { cocaine } \\
\text { consumption }\end{array}$ & $\begin{array}{l}\text { M, C, P, } \\
\text { MA }\end{array}$ & Bimonthly \\
\hline 5 & 72 & 3 & 3 & $\begin{array}{l}\text { Complete remission } \\
\text { on therapy }\end{array}$ & $\begin{array}{l}\text { M, C, P, } \\
\text { MA }\end{array}$ & Bimonthly \\
\hline 6 & 86 & 0 & 3 & Last evaluation & $\mathrm{P}_{1}$ & Daily \\
\hline
\end{tabular}

$I V$ intravenous, $M_{0}$ methylprednisolone boluses of $1 \mathrm{~g} \mathrm{IV} \mathrm{q} 24 \mathrm{~h}$ for three doses, $P_{0}$ prednisone $75 \mathrm{mg}$ (per os, PO) q24 h, $A_{0}$ azathioprine $50 \mathrm{mg}$ PO q24 h, $M$ methylprednisolone $500 \mathrm{mg}$ IV, $C$ cyclophosphamide $500 \mathrm{mg}$ IV, $P$ prednisone $50 \mathrm{mg}$ PO q24 h, $A$ azathioprine $150 \mathrm{mg}$ PO q24 h, $M A$ mycophenolic acid $500 \mathrm{mg}$ PO q6 h, $P_{1}$ prednisone $25 \mathrm{mg}$ PO q24 h

administration; furthermore, bolus administration allows a reduction in long-term maintenance corticosteroid doses and their inherent side-effects due to chronic use. However, these benefits remain theoretical, as bolus therapy has not been demonstrated to have any added value over conventional therapy [10]. Due to the chronicity of PV, adverse effects are frequently associated with use of these corticosteroids. Use of adjuvant therapy (e.g., rituximab, azathioprine, cyclophosphamide, and mycophenolate) increases the efficacy of glucocorticoids while decreasing the effective dose $[6,8]$.

$\mathrm{PV}$ is a chronic, relapsing-remitting autoimmune disorder. Full disease remission is difficult to achieve in PV, having variable clinical outcomes, often determined by the individual's characteristics and environment. According to Murrell and colleagues [6, Table 4], the following considerations are important for PV monitoring : (1) control of disease activity, (2) end of consolidation phase, (3) complete remission on 


\section{A Total body surface with active lesions}

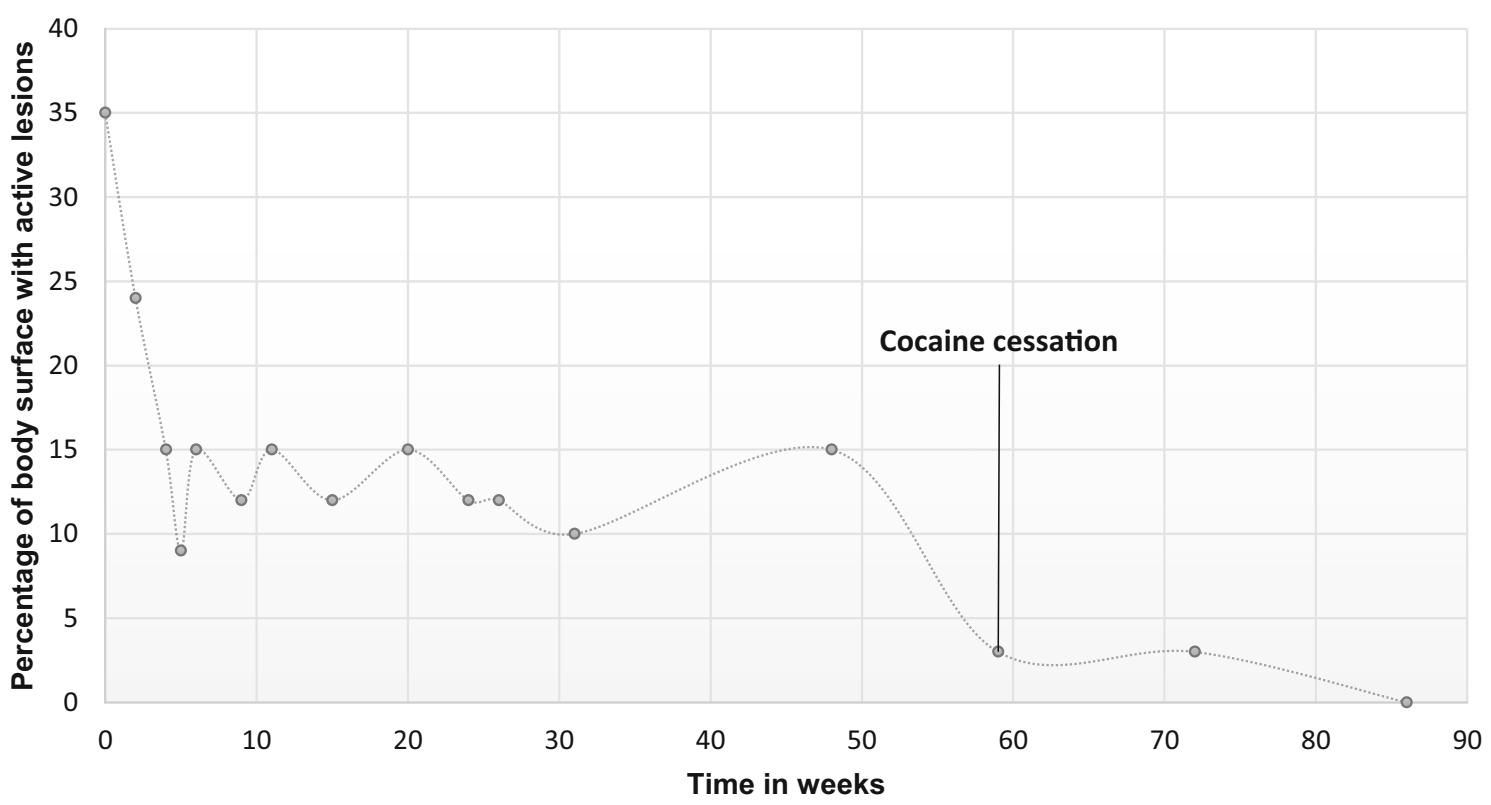

B Clinical evolution through severity index

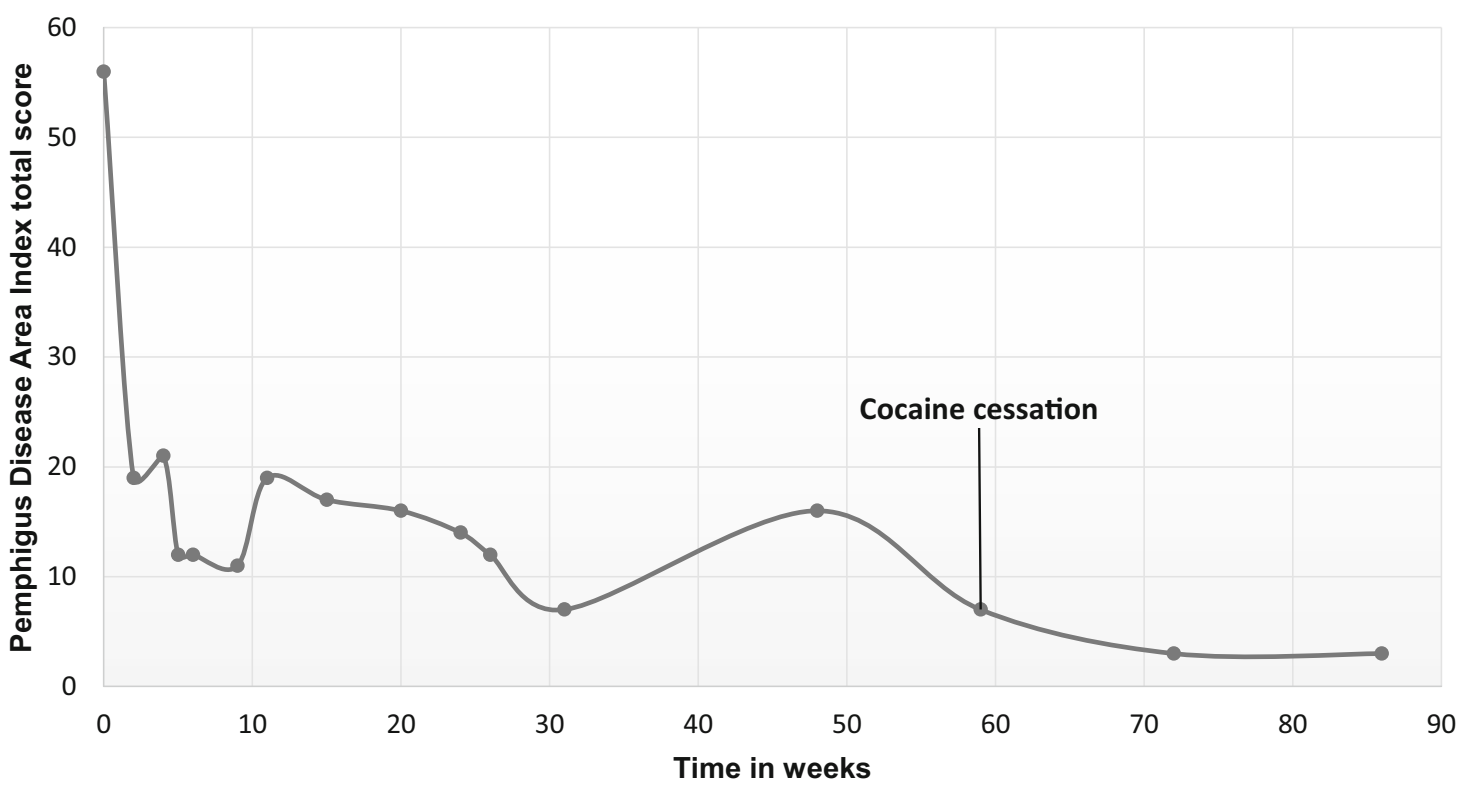

Fig. 3 Graphical representation of clinical evolution. Total body surface with active lesions. $X$-axis, time in weeks. $Y$-axis, percentage of body surface with active lesions

therapy, (4) complete remission off therapy, (5) relapse/flare, and (6) minimal therapy. We decided to use the PDAI to track the clinical evolution and response to the therapeutic management of the patient. The PDAI was found to better assess true clinical severity and was more reproducible than the Autoimmune Bullous Skin Disorder Intensity Score (ABSIS) [11]. In patients with moderate to severe PV, the PDAI has been able to detect small differences in 

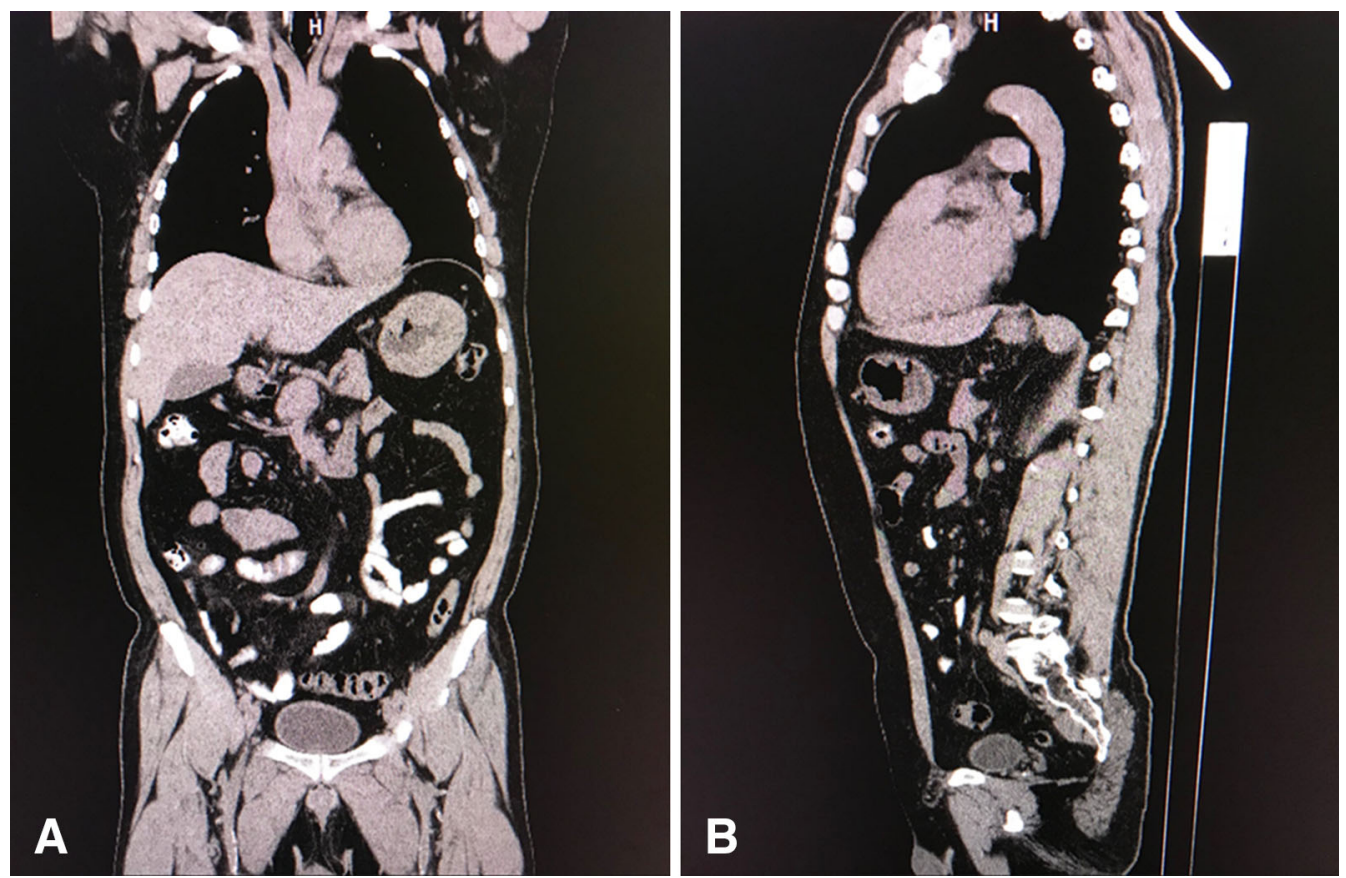

Fig. 4 Thoracoabdominal computerized tomography (CT) scan without contrast. No evidence of significant alterations
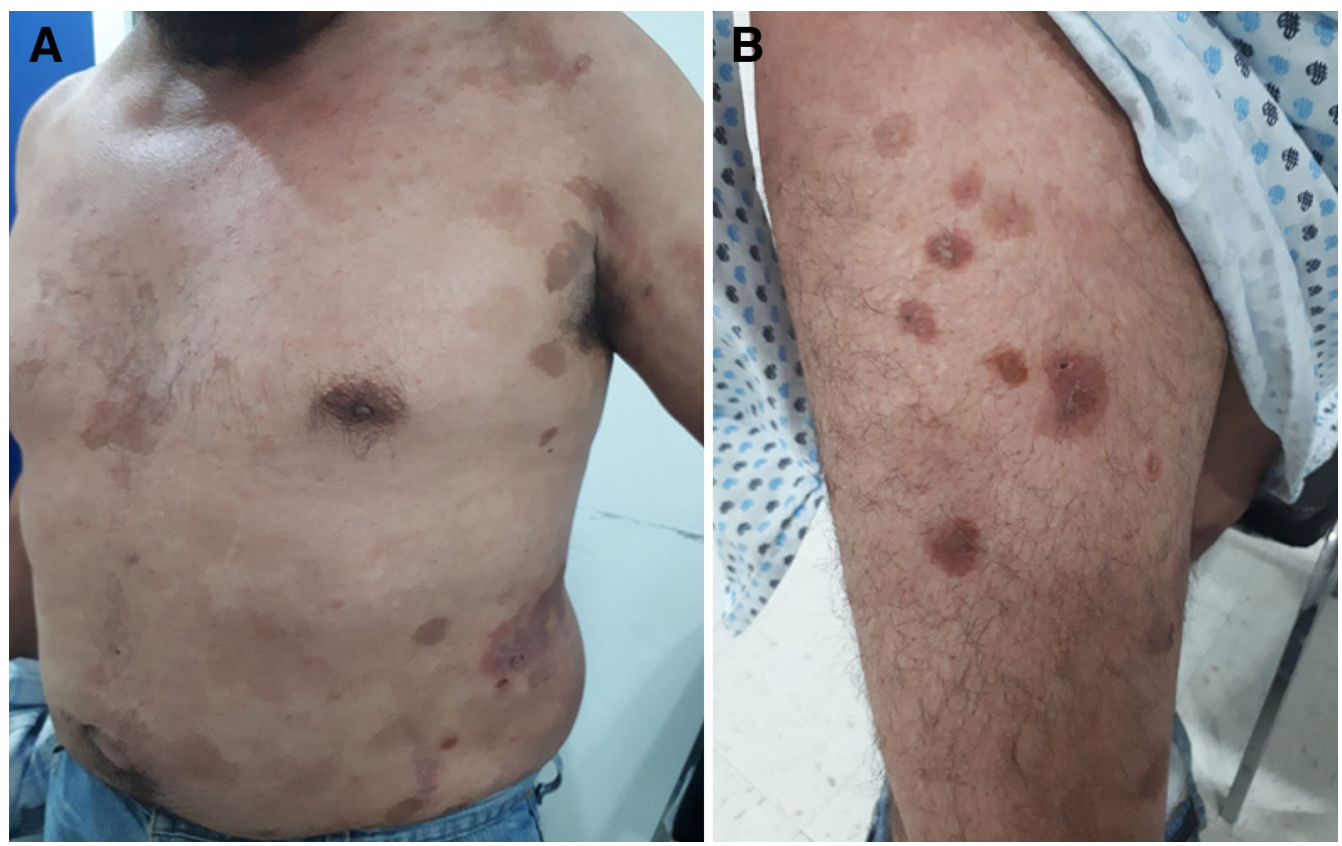

Fig. 5 a Photograph of anterior thorax and abdomen. Posttreatment residual lesions in thoracic and abdominal regions. b Photograph of right thigh. Scabs covering lesions, with residual spots

severity changes [11]. The second phase of therapeutic management, in which standard therapy was provided with methylprednisolone
$500 \mathrm{mg}$ IV, prednisone $50 \mathrm{mg}$ PO, and azathioprine $150 \mathrm{mg} \mathrm{PO}$, served as an adjustment phase. Since the patient experienced his first 
relapse during this second phase, the third phase was implemented after a month. The third phase included methylprednisolone $500 \mathrm{mg}$ IV and cyclophosphamide $500 \mathrm{mg}$ IV once a month, along with maintaining the oral doses of prednisone and azathioprine. Due to the pattern of relapse occurrence, the fourth therapeutic management scheme was implemented. The fourth phase was characterized by maintaining the same doses of oral prednisone and azathioprine, but the frequency of the IV medications (i.e., methylprednisolone $500 \mathrm{mg}$ IV and cyclophosphamide $500 \mathrm{mg}$ IV) was cut in half to bimonthly. The patient did not experience remission, although his cocaine consumption was maintained for almost 4 months. The fifth phase was initiated after the tenth and final relapse. In this phase, azathioprine was replaced by mycophenolic acid $500 \mathrm{mg}$ q6 $\mathrm{h}$ PO. This decision was based on the study of Enk and Knop [12], in which 12 patients with PV had relapses and were managed with azathioprine (1.5-2 $\mathrm{mg} / \mathrm{kg}$ ) and prednisolone $(2 \mathrm{mg} / \mathrm{kg})$, but 11 patients improved clinically when azathioprine was replaced by mycophenolic acid (i.e., $2 \mathrm{~g}$ /day). After 6 months relapse free on therapy with mycophenolic acid PO, prednisone PO, methylprednisolone IV, and cyclophosphamide IV bimonthly, the sixth phase was initiated. After clinical improvement, a risk-benefit assessment was carried out, and it was decided that the patient could be progressed to a maintenance therapy until complete remission off therapy could be initiated. The maintenance therapy established was prednisone $25 \mathrm{mg} \mathrm{PO}$ $\mathrm{q} 24 \mathrm{~h}$, and to date of manuscript preparation (i.e., 3 months), the patient was relapse free.

An additional objective of PV management is to limit use of drugs with side-effects (e.g., glucocorticoids, mycophenolic acid) until the conclusion of therapeutic management or a minimum effective dose is reached $[2,6]$. Treatment time for pemphigus is variable, with duration ranging from 1.5 to 20 years [13]. The first-line treatment in pemphigus is use of systemic corticosteroids such as prednisone at dosage of 0.5 to $1.5 \mathrm{mg} / \mathrm{kg} /$ day. Systemic corticosteroids (e.g., oral or intravenous pulses) can be combined with immunosuppressive adjuvant treatment from the beginning of management; furthermore, this clinical decision should be considered in cases with increased risk of complications due to prolonged use of steroids (i.e. $>4$ months) or the need for continuous use of dosages above $10 \mathrm{mg} /$ day [6]. However, there is limited evidence that addition of adjuvant immunosuppressive agents is superior to treatment with corticosteroids alone [6].

\section{LIMITATIONS AND OTHER CONSIDERATIONS}

One of the limitations of this case report is the completeness of the patient's diagnosis. The patient was diagnosed based on the clinical picture and skin biopsy (i.e., presence of suprabasal bullous lesions with acantholysis). Tissue immunofluorescence microscopy or serological detection of autoantibodies against desmosome proteins was not possible due to lack of availability of laboratory reagents. Durdu and colleagues [14] reported presence of acantholytic cells in $100 \%$ of cytological samples from patients with pemphigus, with specificity of $43 \%$ for PV in a sample of 400 patients. Presence of IgG autoantibodies that bind to the surface of keratinocytes or desmoglein is the gold standard for pemphigus diagnosis and serves for its differentiation from other vesiculobullous or pustular diseases $[15,16]$.

Another limitation of this study is the level of certainty regarding adherence to therapy by the patient. Although the patient reported good adherence to the oral medication and did not miss any of his scheduled IV treatments, he did continue his cocaine consumption. Since our hospital does not have laboratory capabilities to perform high-performance liquid chromatography, concentration levels of prednisone, prednisolone, azathioprine, cyclophosphamide, and mycophenolic acid in plasma, whole blood, urine or bound-to-plasma proteins were not quantified. Furthermore, cocaine metabolite serum levels were not available to monitor the patient's relapses (due to the resource-constrained environment in which our dermatology clinic operates). In an attempt to associate 
the disease activity and cocaine consumption, a surrogate proxy for metabolite serum levels was used in this case report (i.e., cocaine metabolite urinalyses). Although urinalysis for cocaine metabolite detection was performed after each visit, as reported in Supplementary Table 2, serum levels of cocaine metabolites would be ideal to provide further insight into the disease activity (e.g., to correlate disease activity with serum levels of cocaine metabolites). Although cocaine metabolites have a blood clearance half-life of $3.6 \mathrm{~h}$ and up to 3 days in urine, both are adequate for clinical detection of cocaine use (i.e., serum sensitivity of $90 \%$ and specificity of $98.4 \%$ and urine sensitivity of $100 \%$ and specificity of $90.6 \%$ ) [17].

The etiologies considered and investigated included immunizations, hormonal therapy, and other medications. The medications that have been associated with pemphigus include penicillamine, captopril, enalapril, cilazapril, fosinopril, ramipril, nonsteroidal antiinflammatory drugs, rifampicin, levodopa, cephalosporins, phenobarbital, interferon, propranolol, and nifedipine [2]. Drug addictions such as cocaine consumption have also been associated with PV [3, 4]. PV associated with cocaine use is not frequent. In the case presented herein, the patient suffered ten relapses. Ngo and colleagues [4] reported a case of PV associated with cocaine use, where clinical improvement was achieved after 18 months of treatment with prednisone $80 \mathrm{mg}$ PO q24 h and mycophenolate mofetil $1.5 \mathrm{~g}$ PO q24 h. Skin lesions associated with cocaine use exhibit a broad clinical spectrum. The most frequent forms and clinical characteristics of dermatological manifestations secondary to cocaine consumption are leukocytoclastic vasculitis and thrombotic vasculopathy [18]; however, in this patient, both histopathological findings were ruled out.

\section{CONCLUSIONS}

It is very difficult to establish the etiology and the triggering factors that influence reactivations in PV. In this case, persistence of cocaine use (as corroborated by the patient and multiple urinalyses) is likely to be the factor triggering lesion reactivation and responsible for the torpid evolution characterized by ten relapses within 1 year. Although the patient's clinical evolution might have been similar if the patient had ceased cocaine consumption earlier, the event (i.e., cocaine consumption cessation or change in therapy) having a greater effect on the clinical improvement cannot be distinguished. Furthermore, the authors do not claim direct causation between cocaine cessation and pemphigus remission, but associate the clinical improvement with both cocaine cessation and the change to mycophenolic acid in the therapeutic management. The patient showed clinical improvement with a combination of methylprednisolone $500 \mathrm{mg}$ IV and cyclophosphamide $500 \mathrm{mg}$ IV every 15 days, along with prednisone $50 \mathrm{mg} \mathrm{PO}$ q24 $\mathrm{h}$ and mycophenolic acid $500 \mathrm{mg}$ PO q6 h. The patient continued without relapses even after establishing maintenance therapy (i.e., prednisone $25 \mathrm{mg}$ PO q24 h). We cannot definitively conclude whether the change from azathioprine to mycophenolic acid after the tenth relapse was the adjuvant medication responsible for the end of the consolidation phase and complete remission on therapy. To date of manuscript preparation, the patient had not achieved complete remission off therapy. This case study could potentially serve as a guide for management of patients who continuously persist with cocaine use, leading to a clinical picture refractory to multiple therapeutic schemes. We definitively encourage discontinuation of the triggering factor, yet cocaine abuse is a complex disease and must be approached through a multidisciplinary group to address both the medical and psychosocial factors.

\section{ACKNOWLEDGEMENTS}

We would like to commend the work of the medical staff (i.e. specialists, medical residents, and nursing staff) of the Internal Medicine Department at Hospital General León.

Funding. This research did not receive any specific grant from funding agencies in the 
commercial, or not-for-profit sectors but this study was supported by the CONACyT (Consejo Nacional de Ciencia y Tecnología) Grant \#440591. No funding or sponsorship was received for the publication of this article.

Authorship. All named authors meet the International Committee of Medical Journal Editors (ICMJE) criteria for authorship for this article, take responsibility for the integrity of the work as a whole, and have given their approval for this version to be published.

Conflict of Interest. The authors (Omar Jiménez-Zarazúa, Andrés Guzmán-Ramírez, Lourdes N. Vélez-Ramírez, Jesús A. López-García, Leticia Casimiro-Guzmán \& Jaime D. Mondragón) have nothing to disclose.

Compliance with Ethics Guidelines. Approval from the ethical committee was not required due to the nature of this case report. Abiding by the 1964 Declaration of Helsinki and its later amendments or comparable ethical standards, patient anonymity was guaranteed. Upon hospital admission, the patient signed an informed consent permitting the use of their clinical file information for didactic and research purposes.

Data Availability. The clinical data supporting the conclusions of this article is included in the article.

Open Access. This article is distributed under the terms of the Creative Commons Attribution-NonCommercial 4.0 International License (http://creativecommons.org/licenses/ by-nc/4.0/), which permits any noncommercial use, distribution, and reproduction in any medium, provided you give appropriate credit to the original author(s) and the source, provide a link to the Creative Commons license, and indicate if changes were made.

\section{REFERENCES}

1. Joly P, Mouquet $\mathrm{H}$, Roujeau JC, et al. A single cycle of rituximab for the treatment of severe pemphigus.
N Engl J Med. 2007;357(6):545-52. https://doi.org/ 10.1056/NEJMoa067752 (PMID: 17687130).

2. Kasperkiewicz M, Ellebrecht CT, Takahashi H, et al. Pemphigus. Nat Rev Dis Primers. 2017;3:17026. https://doi.org/10.1038/nrdp.2017.26 (PMID: 28492232).

3. Laguna C, Sánchez-Carazo JL, Pérez-Ferriols A, et al. Pemphigus vulgaris associated with cocaine snorting. J Eur Acad Dermatol Venereol. 2008;22(5):645-6. https://doi.org/10.1111/j.14683083.2007.02550.x (PMID: 18266690).

4. Ngo JT, Trotter MJ, Robertson LH. Pemphigus vegetans associated with intranasal cocaine abuse. J Cutan Med Surg. 2012;16(5):344-9. https://doi. org/10.1177/120347541201600512 22971310).

(PMID:

5. Ruocco V, Ruocco E, Schiavo AL, et al. Pemphigus: etiology, pathogenesis, and inducing or triggering factors: facts and controversies. Clin Dermatol. 2013;31(4):374-81. https://doi.org/10.1016/j. clindermatol.2013.01.004 (PMID: 23806154).

6. Murrell DF, Peña S, Joly P, et al. Diagnosis and management of pemphigus: recommendations by an international panel of experts. J Am Acad Dermatol. 2018. https://doi.org/10.1016/j.jaad.2018. 02.021 (PMID: 29438767).

7. Guillen S, Khachemoune A. Pemphigus vulgaris: a short review for the practitioner. Dermatol Nurs. 2007;19(3):269-72 (PMID: 17626506).

8. Atzmony L, Hodak E, Leshem YA, et al. The role of adjuvant therapy in pemphigus: a systematic review and meta-analysis. J Am Acad Dermatol. 2015;73(2):264-71. https://doi.org/10.1016/j.jaad. 2015.04.038 (PMID: 26088689).

9. Frew JW, Martin LK, Murrel DF. Evidence-based treatments in pemphigus vulgari and pemphigus foliaceus. Dermatol Clin. 2011;29(4):599-606. https://doi.org/10.1016/j.det.2011.07.001 (PMID: 21925004).

10. Harman KE, Brown D, Exton LS, et al. British Association of Dermatologists' guidelines for the management of pemphigus vulgaris 2017. B Br J Dermatol. 2017;177(5):1170-201. https://doi.org/ 10.1111/bjd.15930 (PMID: 29192996).

11. Rosenbach M, Murrell DF, Bystryn JC, et al. Reliability and convergent validity of two outcome instruments for pemphigus. J Invest Dermatol. 2009;129(10):2404-10. https://doi.org/10.1038/jid. 2009.72 (Epub 2009 Apr 9 PMID: 19357707).

12. Enk AH, Knop J. Mycophenolate is effective in the treatment of pemphigus vulgaris. Arch Dermatol. 
1999;135(1):54-6. archderm.135.1.54 (PMID: 9923781).

13. Cholera M, Chainani-Wu N. Management of pemphigus vulgaris. Adv Ther. 2016;33(6):910-58. https://doi.org/10.1016/j.clindermatol.2011.03.014 (PMID: 27287854).

14. Durdu M, Baba M, Seçkin D. The value of Tzanck smear test in diagnosis of erosive, vesicular, bullous, and pustular skin lesions. J Am Acad Dermatol. 2008;59(6):958-64. https://doi.org/10.1016/j.jaad. 2008.07.059 (PMID: 18929431).

15. Santoro FA, Stoopler ET, Werth VP. Pemphigus. Dent Clin North Am. 2013;57:597-610. https://doi. org/10.1016/j.cden.2013.06.002

(PMID: 24034068).

16. Stanley JR, Amagai M. Pemphigus, bullous impetigo, and the staphylococcal scalded-skin syndrome. N Engl J Med. 2006;355(17):1800-10. https://doi. org/10.1056/NEJMra061111 (PMID: 17065642).
17. Linder MW, Bosse GM, Henderson MT, et al. Detection of cocaine metabolite in serum and urine: frequency and correlation with medical diagnosis. Clin Chim Acta. 2000;295(1-2):179-85 (PMID: 10767403).

18. Imbernón-Moya A, Chico R, Aguilar-Martínez A. Cutaneous and mucosal manifestations associated with cocaine use. Med Clin (Barc). 2016;146(12):544-9. https://doi.org/10.1016/j. medcli.2016.02.015 (Epub 2016 Mar 28 PMID: 27033438).

19. Eskin-Schwartz M, David M, Mimouni D. Mycophenolate mofetil for the management of autoimmune bullous diseases. Immunol Allergy Clin North Am. 2012;32(2):309-15. https://doi.org/ 10.1016/j.iac.2012.04.010 (PMID: 22560143).

20. Kasperkiewicz M, Schmidt E, Zillikens D. Current therapy of the pemphigus group. Clin Dermatol. 2012;30(1):84-94. https://doi.org/10.1016/j. clindermatol.2011.03.014 (PMID: 22137231). 